10. M. Thomm and G. Wich, ibid. 16, 151 (1988); W.-D. Reiter, U. Hüdepohl, W. Zillig, Proc. Natl. Acad. Sci. U.S.A. 87, 9509 (1990); W. Hausner, G. Frey, M. Thomm, J. Mol. Biol. 222, 495 (1991); J. Hain, W.-D. Reiter, U. Hüdepohl, W. Zillig, Nucleic Acids Res. 20, 5423 (1992); U. Hüdepohl, W.-D. Reiter, W. Zillig, Proc. Natl. Acad. Sci. U.S.A. 87, 5851 (1990).

11. R. J. White and S. P. Jackson, Cell 71, 1041 (1992).

12. D. B. Nikolov et al., Nature 360, 40 (1992); D. I. Chasman, K. M. Flaherty, P. A. Sharp, R. D. Kornberg, Proc. Natl. Acad. Sci. U.S.A. 90, 8174 (1993).

13. Y. Kim, J. H. Geiger, S. Hahn, P. B. Sigler, Nature 365, 512 (1993); J. L. Kim, D. B. Nikolov, S. K. Burley, ibid., p. 520; S. Burley, personal communication.

14. R. Creti et al., J. Mol. Evol. 33, 332 (1991)

15. N. Nakajima, M. Horikoshi, R. G. Roeder, Mol. Cell. Biol. 8, 4028 (1988); R. J. White, S. P. Jackson, P. W. J. Rigby, Proc. Natl. Acad. Sci. U.S.A. 89, 1949 (1992)

16. S. Buratowski, S. Hahn, L. Guarente, P. A. Sharp, Cell 56, 549 (1989); E. Maldonado, I. Ha, P. Cortes, L. Weis, D. Reinberg, Mol. Cell. Biol. 10, 6335 (1990); K. Hisatake, R. G. Roeder, M. Horikoshi, Nature 363, 744 (1993)

17. S. Buratowski and H. Zhou, Proc. Natl. Acad. Sci. U.S.A. 90, 5633 (1993)
18. Cell 71, 221 (1992); T. Colbert and S. Hahn, Genes Dev. 6, 1940 (1992); A. Lopez-de Leon, M. Librizzi, K. Tuglia, I. Willis, Cell 71, 211 (1992).

19. R. J. White, P. Baumann, S. P. Jackson, unpublished results.

20. Abbreviations for the amino acid residues are as follows: A, Ala; C, Cys; D, Asp; E, Glu; F, Phe; G, Gly; H, His; I, Ile; K, Lys; L, Leu; M, Met; N, Asn; P Pro; Q, Gln; R, Arg; S, Ser; T, Thr; V, Val; W, Trp; and $Y, T y r$.

21. R. Janknecht et al., Proc. Natl. Acad. Sci. U.S.A. 88, 8972 (1991).

22. A. J. Bannister, T. M. Gottlieb, T. Kouzarides, S. P. Jackson, Nucleic Acids Res. 21, 1289 (1993).

23. We are indebted to $P$. Cammarano for providing archaebacterial genomic DNAs; to N. Finnie, A. Bannister, and T. Kouzarides for the GST protein derivatives; to R. Weinzierl and R. Tjian for the TBP monoclonal antibody 16E8; and to G. Ghosh and $P$. Sigler for the $P$. woesei TFB expression plasmid. We also thank $M$. McAndrew and $J$. Hyde for advice on PCR conditions, R. Dendy for secretarial assistance, and members of S.P.J.'s laboratory for advice and encouragement. The laboratory of S.P.J. is funded principally by project grant SP2143/0101 from the Cancer Research Campaign (UK)

19 October 1993; accepted 29 April 1994

\title{
Candidate Gene Associated with a Mutation Causing Recessive Polycystic Kidney Disease in Mice
}

\section{Judith H. Moyer, Monica J. Lee-Tischler, Heajoon-Y. Kwon, Jeffrey J. Schrick, Ellis D. Avner, William E. Sweeney, Virginia L. Godfrey, Nestor L. A. Cacheiro, J. Erby Wilkinson, Richard P. Woychik*}

A line of transgenic mice was generated that contains an insertional mutation causing a phenotype similar to human autosomal recessive polycystic kidney disease. Homozygotes displayed a complex phenotype that included bilateral polycystic kidneys and an unusual liver lesion. The mutant locus was cloned and characterized through use of the transgene as a molecular marker. Additionally, a candidate polycystic kidney disease (PKD) gene was identified whose structure and expression are directly associated with the mutant locus. A complementary DNA derived from this gene predicted a peptide containing a motif that was originally identified in several genes involved in cell cycle control.

Autosomal recessive PKD (ARPKD) typically affects infants and children at an estimated incidence of up to 1 in 10,000 live births (1), although prenatal deaths and stillbirths caused by ARPKD may increase that incidence by 27 times (2). One notable difference between recessive and

J. H. Moyer, M. J. Lee-Tischler, H.-Y. Kwon, J. J. Schrick, University of Tennessee Graduate School of Biomedical Sciences, Biology Division, Oak Ridge National Laboratory, Oak Ridge, TN 37831-8077, USA

E. D. Avner and W. E. Sweeney, Department of Pediatrics, University of Washington, Seattle, WA 98105 USA.

V. L. Godfrey, N. L. A. Cacheiro, R. P. Woychik, Biology Division, Oak Ridge National Laboratory, Oak Ridge, TN 37831-8077, USA.

J. E. Wilkinson, Department of Pathobiology, University of Tennessee, Knoxville, TN 37920, USA.

*To whom correspondence should be addressed. dominant PKD (ADPKD), which affects up to 1 in 500 individuals (1), is the nearly invariable presence in the former of noncystic lesions of the liver, ranging from biliary dysplasia to portal fibrosis (3).

Efforts to identify the human chromosomal regions associated with PKD are currently underway. The locus responsible for more than $85 \%$ of ADPKD (PKD1) has been localized to human 16p13.3 (4), and non-PKD1 forms of ADPKD have been linked to additional chromosomes (5). The entire PKD1 locus has been isolated on a series of overlapping clones (6), and although at least 23 genes have been identified in this $750-\mathrm{kb}$ region (7), it still remains unclear which of these genes, if any, is associated with ADPKD.

Animal models of PKD that resembles the various forms of human PKD have been extremely useful for studying the pathogenesis of cystic kidney disease. The recessive cpk (congenital polycystic kidneys) mutation (8) has been examined in considerable detail $(9,10)$. More recently, additional spontaneous recessive mutations in micebpk (BALB/c polycystic kidneys) (11), pcy (polycystic kidneys) (12), and jck (juvenile cystic kidneys) (13) - have been reported. The cystic disease that develops in the $c p k$, $b p k$, and jck models shows similarities to clinical features of human ARPKD, but the dual hepatorenal pathology that is a hallmark of ARPKD has been reported as a consistent feature only in the bpk mouse (11). The cpk locus has been mapped to a region of mouse chromosome 12 with homology to human chromosome 2p24-2p25 (14), and the pcy locus to a region of mouse chromosome 9 homologous to human chromosome 3 (12). In none of these models has the mutant gene been identified, primarily because of a lack of DNA probes directly associated with the locus. Therefore, at present it is impossible to test directly whether the gene associated with any of these mutations corresponds to a gene in humans that causes ARPKD.

Here we report the molecular, genetic, and phenotypic characterization of an insertional mutation in mice that gives rise to a recessive form of PKD that has marked similarities to human ARPKD. Unlike other mutations giving rise to cystic kidneys in mice, this mutation arose by insertional mutagenesis and is called TgN(Imorpk)737Rpw (Imorpk: insertional mutation, Oak Ridge polycystic kidneys) [named in accordance with the new nomenclatural rules for transgenic mice as described (15), and abbreviated here as TgN737Rpw]. The TgN737Rpw mutation arose as part of a large-scale insertional mutagenesis program at the Oak Ridge National Laboratory. The TgN737Rpw transgenic line was generated on the FVB/N inbred genetic background by pronuclear microinjection (16) of the linearized pPyF9-1 construct, which contains the bacterial chloramphenicol acetyltransferase gene under the control of a mutated version of the polyoma early region promoter (17).

The abnormal phenotype arose in the TgN737Rpw line only among transgenic homozygotes, derived from crosses between animals heterozygous for the transgene. In the FVB/N inbred genetic background, external examination revealed that mutant animals have scruffy fur, are severely growth retarded, and have preaxial polydactyly on all limbs. In an analysis of 159 live offspring from a cross of $(\mathrm{C} 3 \mathrm{H} \times \mathrm{FVB} / \mathrm{N})$ TgN737Rpw heterozygotes, mice from 33 complete litters were genotyped for the presence of the transgene. Thirty-five 
(22\%) animals were wild type, 35 were homozygous (22\%), and 89 (56\%) were heterozygous for the transgene, demonstrating expected Mendelian frequencies and the absence of an association of prenatal homozygous lethality with inheritance of the transgene. Essentially equal numbers of male $(18 / 35)$ and female (17/35) mutants were observed. Animals heterozygous for the transgene in the TgN737Rpw line were indistinguishable from their wild-type littermates. The phenotype in homozygotes appeared to be $100 \%$ penetrant because all 113 animals identified by genotypic analysis as transgenic homozygotes were similarly affected. The fact that a detectable mutant phenotype was expressed only by offspring homozygous for the transgene, and not by heterozygotes from either the TgN737Rpw line or any of the other 67 transgenic lines produced with the same transgene construct, suggests that the mutant phenotype in the TgN737Rpw line arose from an insertional mutation caused by the integration of the transgene, and not from an intrinsic phenotypic effect of the pPyF9-1 transgene.

Internal examination of homozygous TgN737Rpw mice on the FVB/N inbred genetic background revealed the following constant traits: bilateral polycystic kidneys and abnormalities associated with the intrahepatic biliary tract. Examination of mutant kidneys revealed that they were slightly enlarged, pale, and contained numerous cysts with concurrent destruction of the surrounding parenchyma (Fig. 1, A and C). The livers were slightly pale with a prominent reticular pattern. As in human ARPKD (18), the pathologic features of the TgN737Rpw phenotype include a spectrum of disease with variable severity in which the primary lesions of the kidneys and liver are expressed to different degrees.

Histologically, the lesions in the kidneys and livers of TgN737Rpw mutant mice were markedly similar to those seen in human ARPKD. The cardinal features of both the kidney lesion (collecting tubule ectasia) and the liver lesion (biliary dysplasia or portal hepatic fibrosis, or both) typical of human ARPKD (18) were a constant finding of the disease in the mutant animals. In the kidneys, an initial mild, multifocal, microscopic dilation of the proximal tubules was followed rapidly by marked dilation and cyst formation of the collecting tubules. Lectin profiling was done as described (11) on a total of 12 (FVB/N)TgN737Rpw mice ranging in age from 1 to 28 days. In newborn animals, all cystic lesions were localized to proximal tubules (Fig. 1I). By postnatal day 7, cystic lesions were detected in collecting tubules (Fig. $1 \mathrm{~J}$ ), and a progressive shift in cyst localization from proximal to collecting tubules was characteristic of all subsequent develop- mental stages. For example, in one animal that survived to day $28,56 \%$ of cysts were localized to collecting tubules. Expansion of the dilated collecting tubules ultimately led to the destruction of the surrounding renal parenchyma (Fig. 1C). This pattern of early proximal tubule dilation followed by a shift to dilation of the distal nephron as the predominant renal lesion is similar to that seen in the $c p k$ and $b p k$ murine models of ARPKD $(10,11)$. Similarly, in human ARPKD, the advanced lesions are localized to the collecting tubules (18), whereas proximal tubular cysts may be demonstrated at early stages of the disease (19).

Abnormalities in the intrahepatic biliary tract involving primarily the bile ducts and ductules represent a constant and prominent feature of both humans with ARPKD and TgN737Rpw mutant mice (Fig. 1, F, $\mathrm{G}$, and $\mathrm{H}$ ). In (FVB/N)-TgN737Rpw mice, the liver lesion is characterized by the proliferation of epithelial cells that arise from the portal triads and form primitive, dysplastic, tortuous ductules that expand the portal and periportal areas (Fig. 1G). The proliferating cells were poorly differentiated and possessed characteristics of oval cells, a cellular population that was initially recognized in experimentally induced models of hepatocellular carcinoma (20). In the livers of some TgN737Rpw homozygotes, these cells disrupted the limiting plate and isolated individual hepatocytes.

To evaluate how genetic background might influence the expression of the TgN737Rpw phenotype, we are in the process of making the mutation congenic on the $\mathrm{C} 3 \mathrm{H}$ inbred genetic background. Most TgN737Rpw mutant mice on the FVB/N background died during the first week of life, although a few animals have lived for
Fig. 1. Gross appearance and histopathology of the kidney and liver of TgN737Rpw mice. (A) Typical gross lesions of bilateral polycystic kidneys in an (FVB/N)-TgN737Rpw mouse. (B) Cross section demonstrating the renal cortex and medulla from a control FVB/N mouse. Staining with hematoxylin and eosin (H\&E); magnification, $\times 12$. (C) Cystic dilation of the collecting ducts in the medulla of the kidney of an (FVB/N)TgN737Rpw mouse. H\&E; $\times 12$. (D) Early lesion in the renal cortex of an (FVB/N)TgN737Rpw mouse demonstrating cystic tubular dilation. H\&E; $\times 30$. (E) Normal liver of an FVB/N control mouse. H\&E; $\times 30$. (F) Hyperplastic and dysplastic bile ductules in the liver of an (FVB/N)-TgN737Rpw mouse. H\&E; $\times 30$. (G) Higher magnification of the portal area of the liver of an (FVB/ N)-TgN737Rpw mouse. Note the expansion of the portal area and the proliferation of small bile ductules. H\&E; $\times 65$. (H) Biliary hyperplasia, dysplasia, and portal fibrosis in the liver of $\begin{array}{llll}\text { an } & (\mathrm{FVB} / \mathrm{N} \quad \mathrm{X} & \mathrm{C} 3 \mathrm{H})-\mathrm{F} 1\end{array}$ TgN737Rpw mouse. Staining with Masson's trichrome; $\times 65$. (I and J) Serial sections of the kidney of a 7-day-old (FVB/N)TgN737Rpw mouse stained with Lotus tetragonobolus agglutinin (I), a marker of proximal tubules, and Dolichos biflorus agglutinin (J), a marker of collecting ducts.
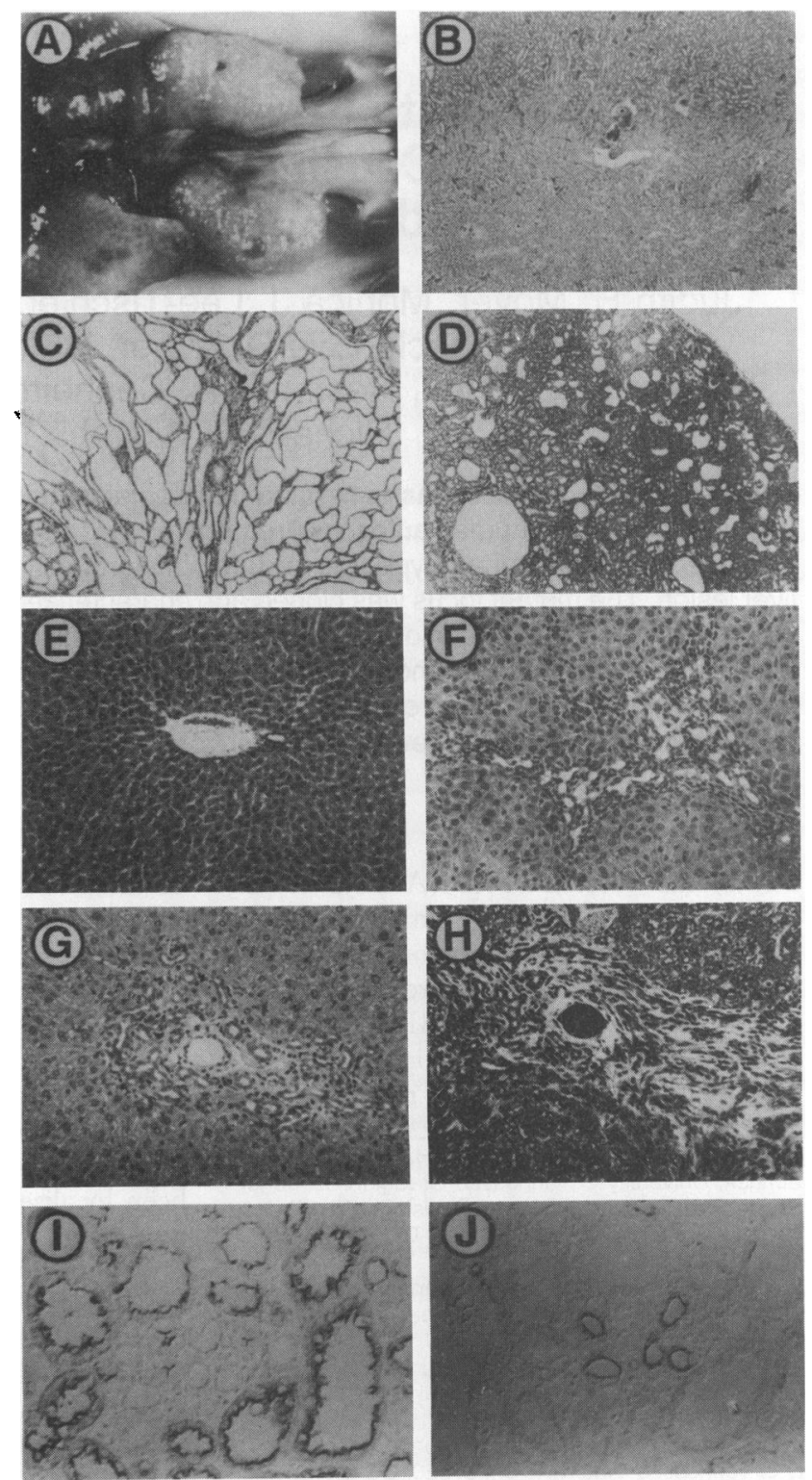
several months. In contrast, $(\mathrm{FVB} / \mathrm{N} \times$ $\mathrm{C} 3 \mathrm{H})-\mathrm{TgN} 737 \mathrm{Rpw}$ mice had a less severe phenotype that was maintained through at least six generations of backcrosses to the $\mathrm{C} 3 \mathrm{H}$ strain. Initial experiments indicated that these animals live longer, have polydactyly that is morphologically more variable and considerably less pronounced in most animals, develop renal cysts at a slower rate, and have a less aggressive liver

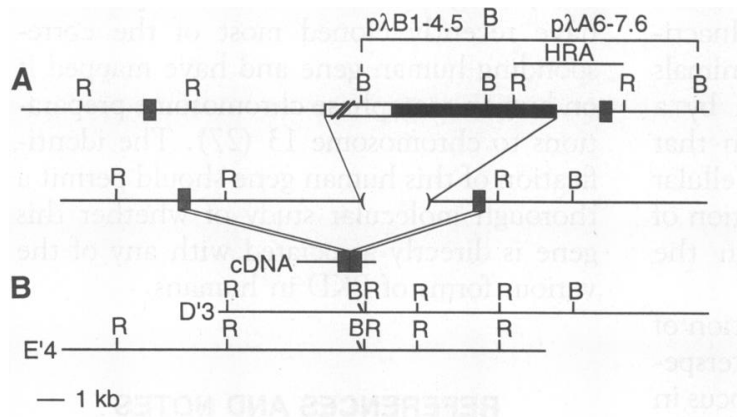

Fig. 2. The TgN737Rpw locus and expression analysis. (A) Mutant TgN737Rpw locus and the corresponding wild-type cDNA, showing the exons from the cloned genomic region. With use of the transgene as a probe, two Bam $\mathrm{HI}$ restriction fragments of $4.5 \mathrm{~kb}$ $(\mathrm{p} \lambda \mathrm{B} 1-4.5)$ and $7.6 \mathrm{~kb}(\mathrm{p} \lambda \mathrm{A6}-7.6)$ were identified at the transgene integration site (black box, transgene sequences; open box, cointegrating DNA) in TgN737Rpw/TgN737Rpw DNA that contained sequences flanking the pPyF9-1 inserted sequences at the $5^{\prime}$ and $3^{\prime}$ junctions, respectively. Both Bam $\mathrm{HI}$ fragments were subsequently cloned and HRA was derived by Eco RI digestion of pגA6-7.6 (21). Approximately 21 $\mathrm{kb}$ of cointegrating DNA (open box) were identified flanking the $5^{\prime}$ end of the transgene sequences. Molecular characterization and comparison of the wild-type and mutant loci revealed that a deletion of no more than $2.77 \mathrm{~kb}$ of genomic DNA (parentheses) occurred at the transgene integration site, but that no exons were deleted. The approximate locations of two exons from the candidate gene, which are located upstream and downstream from the integration site and are adjacent to each other on the cDNA, are indicated (shaded boxes). B, Bam HI; R, Eco RI. (B) Overlapping clones ( $D^{\prime} 3$ and $E^{\prime} 4$ ) were obtained by screening of a wild-type FVB/N lambda library with HRA (21). The clones are aligned with respect to the corresponding mutant locus (A). (C) Southern analysis showing restriction fragment length polymorphism between wild-type and transgenic mice. A Southern blot containing Eco RI-digested genomic DNA from wild-type $(+1+)$, TgN737Rpw/ $\operatorname{TgN737Rpw}(\mathrm{Tg} / \mathrm{Tg})$, and TgN737Rpw/+ $(\mathrm{Tg} /+)$ mice was hybridized with HRA. A 3.0-kb wild-type Eco RI fragment and a 4.2-kb mutant fragment are observed when hybridized with the radioactively labeled HRA probe. Molecular sizes are shown on the left (in kilobases). (D) TgN737Rpw gene expression. Lane 1, mutant kidney; lane 2, mutant liver; lane 3, wild-type kidney; and lane 4, wild-type liver, with radioactively labeled CDNA5 as the hybridization probe on poly (A) ${ }^{+}$-selected RNA (21). RNA molecular size standards and the position of the $28 S$ ribosomal subunit are indicated on the left. The blot was subsequently hybridized with a chick tubulin probe, and results are shown in the lower panel.

Fig. 3. Predicted amino acid sequence of CDNA5 and identification of tetratricopeptide repeat (TPR) domains. A 2472-nucleotide open reading frame beginning with the second in-frame ATG predicts a peptide that is 824 amino acids in length. The second ATG was chosen as the putative start site because it is in better agreement with the Kozak consensus (31). The cDNA nucleotide sequence has been deposited with GenBank (accession number L31959). The 10 TPR domains in the TgN737Rpw gene are underlined. TPRs $1,2,3$, and 4 are separated by 2,5 , and 145 amino acids, respectively, whereas the repeats in the remaining TPRs are uninterrupted.

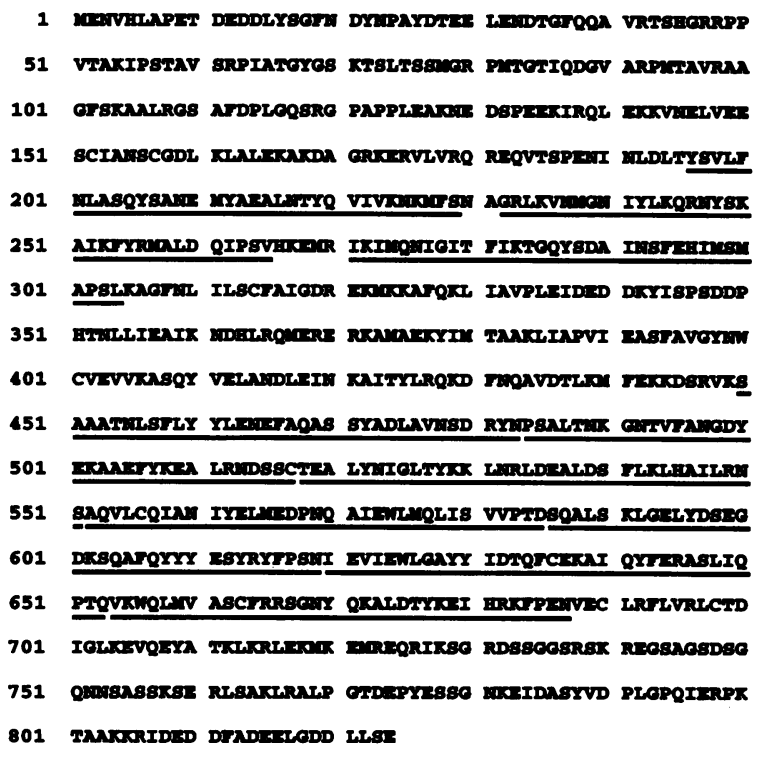

SCIENCE • VOL. 264 • 27 MAY 1994 lesion. Although the kidney lesions were morphologically similar in these backcrossed TgN737Rpw mice, with cysts being localized to collecting tubules by postnatal day 70 , the liver lesions were characterized by biliary hyperplasia, dysplasia, and portal fibrosis (Fig. 1H). The significant difference in the lesions when the TgN737Rpw gene is on a different genetic background suggests the presence of important modifier genes. This may also be the case in human ARPKD, in which the severity of the phenotype and the nature of the liver lesion can be quite variable (18).

For characterization of the mutant locus at the molecular level, several kilobases of DNA flanking the transgene insertion site were isolated through the use of a transgene-derived probe, cloned, and characterized (Fig. 2, A and B) (21). For confirmation that the isolated clones contained genomic sequences flanking the insertion site, a probe corresponding to the region adjacent to the transgene integration site was derived from one clone and used in Southern (DNA) analysis to identify restriction fragment length polymorphisms between wild-type and mutant DNA (Fig. 2C). Detailed restriction mapping, Southern hybridization, and sequence analyses of the cloned region (22) revealed that no more than $2.77 \mathrm{~kb}$ of host genomic DNA were deleted at the transgene integration site (Fig. 2A) and that approximately $21 \mathrm{~kb}$ of mouse genomic DNA had cointegrated along with transgene sequences (Fig. 2A). Pulsed-field gel electrophoresis analysis (22) yielded no evidence for major structural alterations at or near the transgene integration site, and no karyotypic changes were evident in metaphase chromosome preparations from heterozygous animals (22). The fact that a very large region surrounding and including the mutant locus did not contain any major structural alterations suggested to us that the insertional mutation in this line was most likely a single gene defect.

To identify a gene whose expression was affected by the insertional mutation, we systematically screened the cloned genomic sequences from the mutant region for the presence of exons, using a cross-species hybridization approach (23). With this and other methods, we ultimately identified two small exons located both upstream and downstream from the transgene insertion site (Fig. 2A). One exon-containing fragment was then used to purify several complementary DNA (cDNA) clones, one of which, called cDNA5, was determined to be $3043 \mathrm{bp}$ in length fincluding a 19-bp polyadenylate [poly(A)] tail $\}$ and to contain a long open reading frame that potentially represents the entire peptide encoded for by the predominant mRNA species (Fig. 3). 
Northern (RNA) analysis revealed that the primary wild-type transcript detected by a radioactively labeled $\mathrm{CDNA} 5$ probe is 3.2 $\mathrm{kb}$ in length (Fig. 2D), which is in good agreement with the size of cDNA5 [the difference in size is likely due to the presence of a poly(A) tail on the mRNA]. Two transcripts of larger molecular size, about 5.4 and $7.0 \mathrm{~kb}$, were identified in all wildtype tissues that were analyzed; these transcripts were substantially less abundant than the 3.2-kb transcript and may represent partially processed precursors of the $3.2-\mathrm{kb}$ transcript. The 3.2-kb transcript was normally expressed in a number of tissues (22), including kidney and liver (Fig. 2D), which are two tissues severely affected in the adult by the mutation. Expression of the $3.2-\mathrm{kb}$ and the $7.0-\mathrm{kb}$ transcripts was abolished in all mutant tissues examined, including the adult kidney and liver (Fig. 2D), from TgN737Rpw homozygotes. Although the nature of the 5.4-kb transcript observed in the samples from the mutant animals is presently unclear, the fact that it is present at slightly higher levels in some mutant tissues has led us to speculate that it represents an aberrantly processed form of transcript that uniquely arises from the mutant allele.

The protein predicted by cDNA5 is unique and has not been previously described in GenBank. Analysis of the primary amino acid sequence and a search with the BLAST network service of the National Center for Biotechnology Information revealed that the protein contains 10 copies of an internally repeated 34-amino acid sequence referred to as the tetratricopeptide repeat (TPR) (24) (Fig. 3). The TPR was first described in lower eukaryotes as a motif associated with several genes involved in cell cycle control (24). More recently, TPRcontaining proteins in yeast and Drosophila have been discovered that function in various cellular processes, including protein import, transcription, and neurogenesis (25). Although most of the TPR proteins characterized to date are located in the nucleus, at least two are associated with the mitochondrial membrane (25). Our finding that the interrupted gene at the mutant locus in the TgN737Rpw line contains a motif common to several genes involved in cell cycle control could suggest that this gene normally functions to regulate the cell cycle in the epithelial component of the kidney. Inactivation of the gene in the mutant animals may cause an epithelial hyperplasia by a perturbation in cell cycle progression that results either in activation of the cellular proliferative response or in the alteration of the normal pattern of apoptosis in the developing kidney.

Finally, through use of a combination of somatic cell hybrids $(22,26)$ and interspecific backcross analyses, the mutant locus in the TgN737Rpw line has been mapped to mouse chromosome 14 between the $\mathrm{T}$ cell receptor $\alpha(T c r \alpha)$ and retinoblastoma ( $R b$ 1) genes (Fig. 4). The recombination frequencies were determined by analysis of pairs of markers in interspecies backcross mice: 6 out of 70 mice had chromosomes recombinant for Tcra and Rb-1 (8.6 \pm 3.35) [centiMorgans $\pm S D$ ], 1 out of 84 mice was recombinant for HRA (see Fig. $2 A)$ and $T c r \alpha(1.19 \pm 1.18)$, and 5 out of 66 mice were recombinant for $R b-1$ and HRA $(7.58 \pm 3.26)$. This chromosomal assignment rules out allelism of TgN737Rpw with pcy and $c p k$, which map to chromosomes 9 and 12 , respectively (12, 14). Moreover, complementation testing with the bpk mouse has ruled out allelism between TgN737Rpw and $b p k$ (22). Therefore, it appears likely that the TgN737Rpw mutation represents a previously uncharacterized PKD mutation in the mouse.

The phenotypes of the various forms of heritable PKD in humans are substantially different. On the basis of the recessive mode of inheritance and disease pathology, the phenotype of the TgN737Rpw mutants most closely resembles ARPKD. Therefore, the TgN737Rpw mutant mouse may represent an animal model for this particular form of human PKD. Unlike the other
Fig. 4. Genetic map of the TgN737Rpw locus on the basis of interspecific backcross analysis. (A) The position of the TgN737Rpw locus on mouse chromosome 14 relative to the T cell receptor $\alpha$ (Tcr $\alpha)$ and retinoblastoma-1 (Rb-1) genes. HRA was used as a probe to identify the TgN737Rpw locus. Distances between loci are indicated on the left (in centiMorgans). (B) Summary of Mus musculus/Mus spretus interspecific backcross analysis results. The segregation pattern places the TgN737Rpw locus on chromosome 14 between the $T c r \alpha$ and $R b-1$ genes. Boxes in columns represent the chromosomal linkage pattern inherited by backcross progeny that could be scored for all three markers. Backcross experiments were done as described (32). The number of mice that inherited a specific haplotype is given at the top of each column. Open boxes represent $M$. musculus alleles; filled boxes represent $M$. spretus alleles.

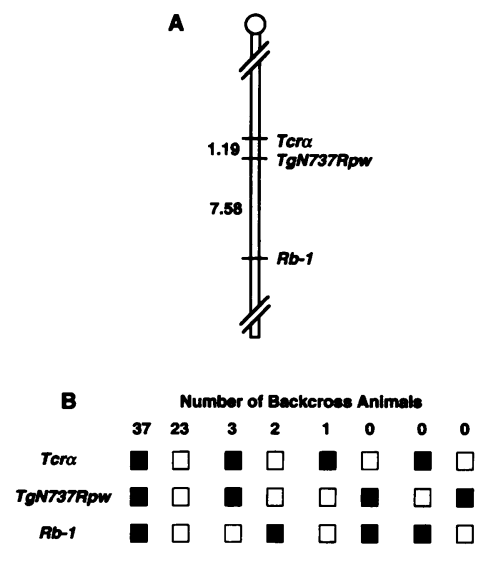

animal models for PKD, the mutant TgN737Rpw locus was tagged with a molecular marker, which has allowed us to clone and characterize a candidate gene whose expression is directly affected by the inserted transgene at the mutant locus. Moreover, using the mouse cDNA as a probe, we have recently cloned most of the corresponding human gene and have mapped it on human metaphase chromosome preparations to chromosome 13 (27). The identification of this human gene should permit a thorough molecular study of whether this gene is directly associated with any of the various forms of PKD in humans.

\section{REFERENCES AND NOTES}

1. B. R. Cole, in The Cystic Kidney, K. D. Gardner and J. Bernstein, Eds. (Kluwer, Dordrecht, Netherlands, 1990), chap. 13.

2. O. Z. Dalgaard, in Diseases of the Kidney, M. B. Strauss and L. G. Welt, Eds. (Little, Brown, Boston, MA, 1971), p. 1252.

3. J. M. Kissane, in Pathology of the Kidney, R. H Heptinstall, Ed. (Little, Brown, Boston, MA, 1974), pp. 69-119.

4. G. G. Germino, S. Somlo, D. Weinstat-Saslow, S. T. Reeders, Kidney Int. 43 (suppl. 39), 20 (1993).

5. S. Nerby and M. Schwartz, Lancet 336,323 (1990); A. F. Wright et al., Human Genet. 90, 569 (1993); W. J. Kimberling, S. Kumar, P. A. Gabow, J. Am. Soc. Nephrol. 4, 816 (1993); M. C. Daoust D. G. Bichet, S. Somlo, ibid., p. 262; D. J. M. Peters et al., Nature Genet. 5, 359 (1993).

6. G. G. Germino et al., Genomics 13, 144 (1992).

7. S. T. Reeders, Nature Genet. 1, 235 (1992).

8. E. S. Russell and E. C. McFarland, Mouse Newslett. 56, 40 (1977).

9. B. D. Cowley, L. J. Chadwick, J. J. Grantham, J. P Calvet, J. Am. Soc. Nephrol. 1, 1048 (1991); E. D Avner, W. E. Sweeney, W. J. Nelson, Proc. Natl. Acad. Sci. U.S.A. 89, 7447 (1992)

10. E. D. Avner et al., Pediatr. Nephrol. 1, 587 (1987).

11. J. Nauta, Y. Ozawa, W. E. Sweeney, J. C. Rutledge, E. D. Avner, ibid. 7, 163 (1993)

12. H. Takahashi et al., J. Am. Soc. Nephrol. 1, 980 (1991).

13. A. Atala, M. R. Freeman, J. Mandell, D. R. Beier, Kidney Int. 43, 1081 (1993).

14. L. M. Guay-Woodford, P. D'Eustachio, G. A. P Bruns, J. Am. Soc. Nephrol. 4, 814 (1993)

15. J. Gordon, ILAR News 34, 45 (1992).

16. J. W. Gordon, G. A. Scangos, D. J. Plotkin, J. A Barbarosa, F. H. Ruddle, Proc. Natl. Acad. Sci. U.S.A. 77, 7380 (1980); B. Hogan, F. Costantini, E Lacy, in Manipulating the Mouse Embryo: A Laboratory Manual (Cold Spring Harbor Laboratory, Cold Spring Harbor, NY, 1986)

17. E. Bohnlein, K. Chowdhury, P. Gruss, Nucleic Acids Res. 13, 4789 (1985).

18. J. Bernstein, in Pediatric Kidney Disease, C. M Edelmann, Ed. (Little, Brown, Boston, MA, 1978), pp. 557-570; J. Bernstein and K. D. Gardner, in Campbells' Urology (Saunders, Philadelphia, PA, 1986), pp. 1760-1818; M. C. Lippert, in Adult and Pediatric Urology (Mosby, Chicago, IL, 1991), pp. 711-743; E. Lieberman et al., Medicine 50, 277 (1971).

19. E. D. Avner and W. E. Sweeney, J. Am. Soc Nephrol. 3, 292 (1992).

20. E. Farber, Cancer Res. 16, 142 (1956)

21. All methods involving the analysis and cloning of specific DNA fragments were as described (28) We cloned $p \lambda B 1-4.5$ and $p \lambda A 6-7.6$ by generating genomic libraries with size-fractionated adult liver DNA from a TgN737Rpw homozygote that had 
been digested to completion with $\mathrm{Bam} \mathrm{HI}$; these libraries were screened with a probe corresponding to sequences within the transgene. The wildtype clones ( $D^{\prime} 3$ and $\left.E^{\prime} 4\right)$ corresponding to the transgene insertion site were cloned by screening of a wild-type FVB/N partial Sau $3 A$-digested genomic lambda library with HRA, an internal Eco RI fragment from pגA6-7.6 that contains sequences flanking the transgene insertion. Total RNA was isolated by use of the guanidinium isothiocyanate procedure (28) and enriched for polyadenylated $\left[\right.$ poly $\left.(A)^{+}\right]$RNA by passage through oligo(dT) columns. The cDNA library was prepared from poly(A) + RNA isolated from whole 14.5 days post-coitum (dpc) mouse embryos: Sma I adaptors and Eco RI ends were added and the double-stranded cDNAs ligated into the $\lambda g t 10$ vector (Stratagene). Hybridization probes were labeled with [ $\alpha-{ }^{32} P$ ]deoxycytidine 5 '-triphosphate (dCTP) by the random-hexamer labeling method (29). The Sanger dideoxynucleotide method was used for all sequencing (30).

22. J. H. Moyer et al., unpublished results.

23. W. McGinnis, C. P. Hart, W. J. Gehring, F. H. Ruddle, Cell 38, 675 (1984)

24. R. S. Sikorski, M. S. Boguski, M. Goebl, P. Hieter, ibid. 60, 307 (1990)

25. M. Goebl and M. Yanagida, Trends Biochem. Sci. 16, 173 (1991); I. Van der Leij, M. M. Franse, Y. Elgersma, B. Distel, H. F. Tabak, Proc. Natl. Acad. Sci. U.S.A. 90, 11782 (1993).

26. R. P. Woychik et al., Proc. Natl. Acad. Sci. U.S.A. 87, 2588 (1990)

27. J. J. Schrick et al., unpublished results.

28. J. Sambrook, E. F. Fritsch, T. Maniatis, in Molecular Cloning: A Laboratory Manual (Cold Spring Harbor Laboratory, Cold Spring Harbor, NY, ed. 2, 1989); F. M. Ausubel et al., in Current Protocols in Molecular Biology (Wiley, New York, 1988).

29. A. P. Feinberg and B. Vogelstein, Anal. Biochem. 137, 266 (1984)
30. F. Sanger, S. Nicklen, A. R. Coulson, Proc. Natl. Acad. Sci. U.S.A. 74, 5463 (1977).

31. M. Kozak, J. Cell Biol. 115, 887 (1991).

32. L. Stubbs et al., Genomics 6, 645 (1990).

33. We gratefully acknowledge $E$. Bohnlein for providing the pPyF9-1 construct, B. Beatty for generating the TgN737Rpw line by pronuclear microinjection, P. D'Eustachio for the mouse $\times$ Chinese hamster somatic cell hybrid DNAs, J. Nadeau for the $R b-1$ probe and the Tcr $\alpha$ CDNA that were used as probes in the interspecific backcross mapping analysis, B. L. Hogan and M. E. Dickinson for the $R b-1$ interspecies backcross hybridization experiment and interspecies backcross DNAs and panels, L. Stubbs for the 14.5-dpc cDNA library, and J. Bernstein and J. Rutledge for slides of human tissues. We also thank S. T. Reeders and L. F. Onuchic for their contribution in the computer analysis of the cDNA sequence, and E. M. Rinchik, R. J. Mural, W. R. Jacobs, J. H Nadeau, and B. L. M. Hogan for critically reading the manuscript. Supported by the Office of Health and Environmental Research, U.S. Department of Energy under contract DE-AC05-84OR21400 with Martin Marietta Energy Systems, Inc. (R.P.W.), the National Institute of Environmental Health Sciences under contract IAG 222Y01-ES-10067 (R.P.W. M.J.L.-T., and H.-Y.K.), National Institute of Diabetes and Digestive and Kidney Diseases grant 1 R01 DK45633-01 (J.E.W. and R.P.W.), Nationa Institute of Child Health and Human Development grant R01 HD25323 (R.P.W.), and by NIH predoctoral National Research Service Award NR06789 01 from the National Center for Nursing Research (J.H.M.). Additional support was provided by NIH grant 5 R01 DK44875 (E.D.A. and W.E.S.). All mice used in this study were handled in accordance with the NIH Guide for the Care and Use of Laboratory Animals.

23 December 1993; accepted 7 April 1994

\title{
Summation and Division by Neurons in Primate Visual Cortex
}

\author{
Matteo Carandini and David J. Heeger
}

Recordings from monkey primary visual cortex (V1) were used to test a model for the visually driven responses of simple cells. According to the model, simple cells compute a linear sum of the responses of lateral geniculate nucleus (LGN) neurons. In addition, each simple cell's linear response is divided by the pooled activity of a large number of other simple cells. The cell membrane performs both operations; synaptic currents are summed and then divided by the total membrane conductance. Current and conductance are decoupled (by a complementary arrangement of excitation and inhibition) so that current depends only on the LGN inputs and conductance depends only on the cortical inputs. Closed form expressions were derived for fitting and interpreting physiological data. The model accurately predicted responses to drifting grating stimuli of various contrasts, orientations, and spatiotemporal frequencies.

Since the pioneering work of Hubel and Wiesel (1), there have been a multitude of physiological experiments that studied the visually driven responses of V1 simple cells. A long-standing view is that a simple cell's response depends on a linear sum, over local space and recently past time, of the intensity values in the stimulus (2). The

M. Carandini, Center for Neural Science, New York University, New York, NY 10003, USA.

D. J. Heeger, Department of Psychology, Stanford University, Stanford, CA 94305, USA. linear model of simple cell physiology is attractive because the response of a linear cell can be completely characterized with a relatively small number of measurements. In addition, the linear model explains the selectivity of simple cells for stimulus position, orientation, and direction of motion.

Unfortunately, the linear model falls short of a complete account of simple cell physiology. According to the linear model, doubling the contrast of a (periodic) drifting grating stimulus would double the re- sponse so one would record twice as many action potentials during each period of stimulation. However, simple cells do not behave this way. First, response amplitude saturates at high contrasts (3); doubling the contrast yields fewer than twice the number of action potentials. Second, response phase advances with contrast (4); when contrast is doubled, the action potentials occur sooner during each period of stimulation.

A third fault with the linear model in regard to simple cells is revealed by tests of superposition. A typical simple cell responds vigorously to its preferred orientation but not at all to a perpendicular orientation. According to the linear model, the response to the superimposed pair of stimuli (preferred plus perpendicular) should equal the response to the preferred stimulus presented alone. In fact, the response to the superimposed pair is about half that predicted (5), a phenomenon known as crossorientation inhibition.

To explain these nonlinear aspects of simple cell responses, we have recently proposed a new model of simple cell responses, called the normalization model (6). This model (Fig. 1) begins with an underlying linear stage. The linear stage is followed by a normalization stage, where each cell's linear response to the stimulus is divided by a quantity proportional to the pooled activity of a large number of other cells. Normalization is a nonlinear operation; one input (a cell's underlying linear response) is divided by another input (the pooled activity of a large number of cells). The effect of normalization is that the response of each cell is rescaled with respect to stimulus contrast.

The normalization model explains a large body of otherwise unexplained physiological phenomena (6). According to the model, a cell's selectivity is attributed to summation (the linear stage) and its nonlinear behavior is attributed to division (the normalization stage). The model explains response amplitude saturation because the divisive suppression increases with stimulus contrast. The model also explains crossorientation inhibition because a given cell is suppressed by many other cells, including those with perpendicular orientation tunings. Until now, however, two problems still remained to be solved. First, there was no explanation for why response phase depends on contrast. Second, there was no explanation for how the summation and division computations might be implemented by cortical neurons.

It is common to characterize the electrical behavior of a cell's membrane with electrical circuits made up of resistors and capacitors (Fig. 2). The input to a cell is a current driven by the synaptic conduc- 